\title{
Motion Processing in the Macaque: Revisited with Functional Magnetic Resonance Imaging
}

\author{
Andreas S. Tolias, ${ }^{1}$ Stelios M. Smirnakis, ${ }^{1,2}$ Mark A. Augath, ${ }^{1}$ Torsten Trinath, ${ }^{1}$ and Nikos K. Logothetis ${ }^{1}$ \\ ${ }^{1}$ Max Planck Institute for Biological Cybernetics, Tuebingen, 72076 Germany, and 2Department of Neurology, \\ Massachusetts General Hospital and Brigham and Women's Hospital, Harvard Medical School, Boston, Massachusetts \\ 02114
}

A great deal is known about the response properties of single neurons processing sensory information. In contrast, less is understood about the collective characteristics of networks of neurons that may underlie sensory capacities of animals. We used functional magnetic resonance imaging to study the emergent properties of populations of neurons processing motion across different brain areas. Using a visual adaptation paradigm, we localized a distributed network of visual areas that process information about the direction of motion as expected from single-cell recording studies. However, we found an apparent discrepancy between the directional signals in certain visual areas as measured with blood oxygenation leveldependent imaging compared with an estimate based on the spiking of single neurons. We propose a hypothesis that may account for this difference based on the postulate that neuronal selectivity is a function of the state of adaptation. Consequently, neurons classically thought to lack information about certain attributes of the visual scene may nevertheless receive and process this information. We further hypothesize that this adaptation-dependent selectivity may arise from intra- or interarea cellular connections, such as feedback from higher areas. This network property may be a universal principle the computational goal of which is to enhance the ability of neurons in earlier visual areas to adapt to statistical regularities of the input and therefore increase their sensitivity to detect changes along these stimulus dimensions.

Key words: fMRl; motion; V1; MT; monkey; adaptation
Substantial progress has been made in characterizing the response properties of single neurons involved in the analysis of motion (Maunsell and Newsome, 1987; Parker and Newsome, 1998). In contrast, little is known about the collective properties of contiguous or distributed networks of neurons that may underlie sensory motion processing and motion perception. To study the global properties of neuronal populations processing information about the direction of motion, we used functional magnetic resonance imaging (fMRI) in anesthetized monkeys. The monkey model has several advantages for carrying out these studies, including the ability to compare imaging with electrophysiological data.

In our experiments we used a visual adaptation paradigm in which continuous presentation of a stimulus results in decreased fMRI responses over time. Then abruptly the stimulus changed, and we measured the induced change in the fMRI signal. This paradigm allowed us to determine with blood oxygenation leveldependent (BOLD) imaging whether local regions of macaque cortex process information about direction of motion (GrillSpector et al., 1999; Kourtzi and Kanwisher, 2000). This would not be possible using a standard experimental paradigm, in which various stimulus conditions are compared with a blank screen

\footnotetext{
Received May 17, 2001; revised July 30, 2001; accepted Aug. 9, 2001.

This study was supported by the Max Planck Society and a National Research Service Award from National Institutes of Health-National Eye Institute to A.S.T. We thank B. Prause for technical assistance and Z. Kourtzi, T. S. Lee, B. Prause, G. Rainer, K. Saleem, A. G. Siapas, N. Sigala, and K. F. Tolias for helpful discussions and reading this manuscript.

Correspondence should be addressed to Andreas S. Tolias, Max Planck Institute for Biological Cybernetics, Spemannstrasse 38, Tuebingen, Germany. E-mail: andreas.tolias@tuebingen.mpg.de.

Copyright (ㄷ) 2001 Society for Neuroscience $\quad 0270-6474 / 01 / 218594-08 \$ 15.00 / 0$
}

condition, because visual areas are composed of heterogeneous populations of neurons selective for a wide range of directions of motion organized at a spatial scale beyond the spatial resolution of standard primate fMRI protocols. It is natural to assume that brain areas that adapt selectively to the direction of motion take part in processing direction of motion information. Using the adaptation paradigm, we found the most direct evidence to date that direction-selective information is reflected in the BOLD signal. In agreement with previous single-cell electrophysiology studies, we localized a distributed network of visual areas that process information about the direction of motion. However, we found a discrepancy between the strength of the directionally selective BOLD signal in early visual areas and the prediction one would make using established facts from macaque single-cell electrophysiology. The simple hypothesis that neuronal selectivity is a function of adaptation can account for this difference.

Finally, the adaptation paradigm enabled us to characterize the dynamics of adaptation of large networks of neurons in the macaque visual cortex, taking advantage of the global coverage afforded by fMRI. The study of adaptation is important because it reflects a fundamental computational principle of the nervous system. The perceptual effects of adaptation to a pattern of motion are well demonstrated by celebrated illusions, such as the waterfall illusion (Wohlgemuth, 1911; Mather et al., 1998). The ability to adapt to the statistics of the environment reduces redundancy and increases the dynamic range available for encoding changes (Barlow, 1972; Smirnakis et al., 1997). Adaptation is ubiquitously expressed in the properties of single neurons throughout the nervous system, where the firing rate of motionsensitive neurons from a wide range of species typically decreases during continuous presentation of a moving stimulus (Barlow and 
Hill, 1963; Oyster et al., 1972; Vautin and Berkley, 1977; von der Heydt et al., 1978; Maddess et al., 1988; Ibbotson et al., 1998; Lisberger and Movshon, 1999).

\section{MATERIALS AND METHODS}

Surgical and anesthesia procedures. Experiments were conducted in four healthy monkeys (Macaca mulatta) weighing $5.5-7 \mathrm{~kg}$. The studies were approved by the local authorities (Regierungspraesidium) and were in full compliance with the guidelines of the European Community (EUVD 86/609/EEC) for the care and use of laboratory animals. Surgical procedures for custom-made plastic head holders, anesthesia protocol during the fMRI imaging, and procedures for positioning the visual stimuli have been described previously (Logothetis et al., 1999).

Visual stimulation. A full-field rotating checkerboard polar pattern stimulus was used for both the visual-localization and visual-adaptation protocols $\left(30^{\circ}\right.$ horizontal $\times 23^{\circ}$ vertical) (see Figs. $\left.1 \mathrm{~A}, 2 \mathrm{~A}\right)$. The spatial frequency of the stimulus was $30^{\circ}$ per cycle in the angular dimension (i.e., every $15^{\circ}$, the intensity of the polar stimulus changes from bright to dark). The temporal frequency was $1 / 6 \mathrm{~Hz}$. The stimulus contrast was $100 \%$. During the visual-localization stimulation protocol (see Fig. 1A), the rotating polar was presented for $48 \mathrm{sec}$ followed by $48 \mathrm{sec}$ of no stimulus. The direction of rotation was reversed every $5 \mathrm{sec}$ between the clockwise and counterclockwise directions (see Fig. 1A). This procedure was repeated four times.

During the visual adaptation protocol, a single stimulation trial began with $30 \mathrm{sec}$ of blank screen condition followed by $200 \mathrm{sec}$ of the rotating polar stimulus, rotating in a single direction either clockwise or counterclockwise. Thereafter the direction of rotation of the polar pattern was reversed, and the polar stimulus was presented for another $100 \mathrm{sec}$. Each stimulation trial ended with the blank screen condition for $46 \mathrm{sec}$. During a single experimental session, $20-30$ such stimulation trials were repeated. Control trials in which the direction of motion remained unchanged throughout the trial were interleaved with these stimulation trials.

Note that at the transition point, where the direction of rotation of the polar reversed, the polar pattern was reset to its original phase, which could be seen as a fast visual transient. Similarly, in the control, the phase of the polar stimulus was also reset to zero $200 \mathrm{sec}$ after the onset of the stimulus. The BOLD signal was not sensitive to this transient (see Figs. $2 C, 3 C, 7 A-C)$.

MRI data collection. Experiments were conducted in a vertical 4.7 tesla scanner with a 40-cm-diameter bore (Biospec 47/40v; Brucker Medical, Ettlingen, Germany). The system had a $50 \mathrm{mT} / \mathrm{m}$ (180 $\mu \mathrm{sec}$ rise time) actively shielded gradient coil (B-GA 26, Brucker Medical) of $26 \mathrm{~cm}$ inner diameter. We used a custom chair and custom system for positioning the monkey within the magnet (Logothetis et al., 1999). During the visual localization experiment, we used multi-shot (eight) gradient echo (GE) echo planar imaging (EPI) using a $128 \times 128$ matrix $\left[1 \times 1 \mathrm{~mm}^{2}\right.$ resolution; slice thickness $2 \mathrm{~mm}$; echo time (TE) $=20 \mathrm{msec}$; repetition time $(\mathrm{TR})=750 \mathrm{msec}$; flip angle $(\mathrm{FA})=40^{\circ}$ ] of the whole brain of the monkeys. Typically 13 horizontal brain slices were acquired within $6 \mathrm{sec}$. This process was repeated eight times ( $48 \mathrm{sec}$ total time) during which the stimulus was displayed. The blank screen period followed, which also lasted $48 \mathrm{sec}$, and the same imaging protocol was applied. Two to three slices were then selected for the adaptation experiment. BOLD activity from these slices was acquired at a higher temporal resolution than before (all selected slices were acquired once every second). MRI data collection was the same as before but with $\mathrm{TR}=250 \mathrm{msec}, \mathrm{FA}=20-25^{\circ}$, and multi-shot (four) GE-recalled EPI images used instead. Anatomical images were acquired using a matrix of $256 \times 256(0.5 \times 0.5 \mathrm{~mm}$ resolution; inversion recovery-rapid acquisition with relaxation enhancement).

MRI data analysis. The MRI data were analyzed off-line using our own software developed in MATLAB. We converted the multi-slice data collected during the visual localization experiments (see Fig. $1 A$ ) into a time series of individual voxels. For each image (matrix of signal intensities at a single point in time for a single brain slice), we applied a two-dimensional spatial convolution kernel (Gaussian kernel with SD half the size of a pixel). Functional maps, such as those presented in Figure $1 B$, were generated by cross-correlating the postconvoluted time course at each voxel with a boxcar model of the stimulus presentation protocol (see Fig. 1A). These functional maps were thresholded to identify the significantly activated voxels. The threshold was set at a value of two SDs above the mean of the distribution of correlation coefficients of a region in the functional map where no brain activity was present $(50 \times 50$ voxels; top right corner of the functional map). In addition, we applied a simple clustering algorithm that eliminated random, spuriously activated voxels. We centered a $4 \times 4$ window on each of the activated voxels. If there were not at least another 10 activated voxels within this window, then the activity of the voxel was defined as spurious and not considered significant. Using this simple clustering algorithm, we achieved good localization of visually activated brain areas illustrated by the consistently focal activation over gray matter (see Fig. $1 C$ ).

The borders between a number of visual areas were marked on the basis of anatomical criteria (Gattass et al., 1981; Desimone and Ungerleider, 1986; Gattass et al., 1988). The time course of the BOLD signal during the adaptation experiments was calculated by considering the voxels that were significantly activated during the visual localization experiment. These time series were normalized for each individual image (matrix of signal intensities at a single point in time for a single brain slice) by dividing by the mean of the top $90 \%$ pixel values of each image (the lowest $10 \%$ generally represented the background of the image, i.e., lay outside the head). The average activity across voxels from each area was then calculated for the adaptation experiments.

The BOLD directional index $(\rho)$ was defined as follows: $\rho=$ reboundresponse/initial-response. The rebound-response was defined as the peak response, after the direction of motion changed, of the filtered activity (digital low-pass Butterworth 8 order filter and cutoff frequency 0.125 $\mathrm{Hz})$ relative to baseline. In this case, baseline was defined as the mean BOLD activity $10 \mathrm{sec}$ before the change in the direction of motion. The initial-response was defined as the peak response, after the initial onset of the stimulus, of the filtered activity (digital low-pass Butterworth 8 order filter and cutoff frequency $0.125 \mathrm{~Hz}$ ) relative to baseline. In this case, baseline was defined as the mean BOLD activity $10 \mathrm{sec}$ before the stimulus onset.

Exponential functions were fitted to analyze the time course of adaptation in different visual areas. The characteristics of adaptation across different visual areas were measured by fitting exponentials of the form:

$$
R(t)=A \times e^{\frac{-t}{\tau}}+C,
$$

where $R$ is the fitted responses at time $t, \tau$ is the time constant of the decaying exponential, and $C$ is the fitted steady-state response. The fitted peak is $A+C$.

\section{RESULTS}

\section{Direction selectivity in the BOLD signal of different visual areas}

We identified visual areas in the macaque brain using a visuallocalization stimulation protocol. This stimulation paradigm consisted of four alternating ON/OFF epochs lasting 48 sec each (Fig. $1 A)$. A rotating polar checkerboard pattern was presented during ON epochs. This stimulus has previously been shown to robustly activate the visual cortex of the anesthetized monkey (Logothetis et al., 1999). Functional maps were constructed by crosscorrelating the observed signal at each voxel with a boxcar waveform model of the experimental paradigm (Fig. 1B) (see Materials and Methods). Activation was seen in areas V1, V2, V3, $\mathrm{V} 3 \mathrm{~A}, \mathrm{~V} 4$, and MT as revealed by superimposing the functional maps on the corresponding anatomical images. For instance, part of the activity in the map in Figure $1 B$ contains area V1 (Fig. $1 C$ ). During the visual-localization stimulation protocol, 13 slices (resolution $1 \times 1 \times 2 \mathrm{~mm}^{3}$ ) covering the entire macaque brain were acquired in $6 \mathrm{sec}$. For the adaptation experiments, two to three selected brain slices were acquired at a higher temporal resolution (Fig. 2A). These slices were chosen on anatomical and functional grounds to include visual areas of the monkey cortex, particularly areas $\mathrm{V} 1$ and MT.

The boundaries of the visual areas that we studied were defined on the basis of anatomical criteria (Gattass et al., 1981, 1988; Desimone and Ungerleider, 1986). Specifically, area V1 was determined to lie on the operculum and within the calcarine sulcus. We considered areas V2 and V3A within the posterior and 
A

Figure 1. Localization of visual areas. A, Visual stimulation protocol for localization of visually responsive areas. A rotating checkerboard polar pattern stimulus was presented; its direction was reversed every $5 \mathrm{sec}$ from clockwise to counterclockwise. The stimulus was shown for $48 \mathrm{sec}$ $(O N)$. The ON-condition was interleaved with an off-condition (blank screen condition), also presented for $48 \mathrm{sec}$. Thirteen axial brain slices were acquired during $6 \mathrm{sec}$, and 8 such consecutive acquisitions were completed during $48 \mathrm{sec}$. Each slice had a field of view of $128 \times 128$ voxels, with each voxel size $1 \times 1 \mathrm{~mm}^{2}$ in plane resolution and $2 \mathrm{~mm}$ slice thickness. During the course of the visual localization protocol, there were four consecutive ON/OFF conditions. $B$, The functional map of BOLD signal for a single brain slice was computed by cross-correlating the time course of the BOLD signal for each individual voxel with a boxcar model of the stimulus protocol (see Materials and Methods). $C$, The statistically thresholded functional map is superimposed on the anatomical image (see Materials and Methods). The voxels with significant activation in area V1 are color coded and located posteriorly.

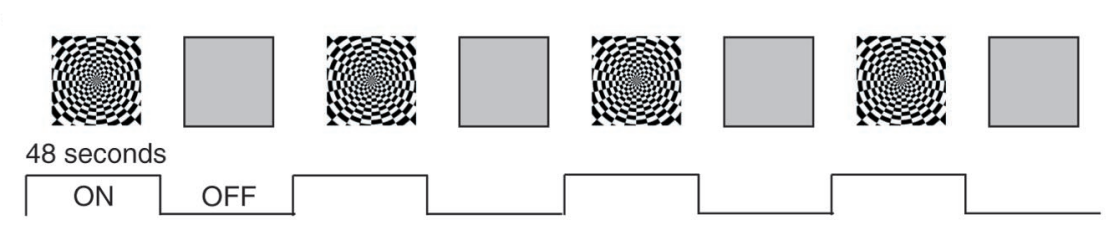

B

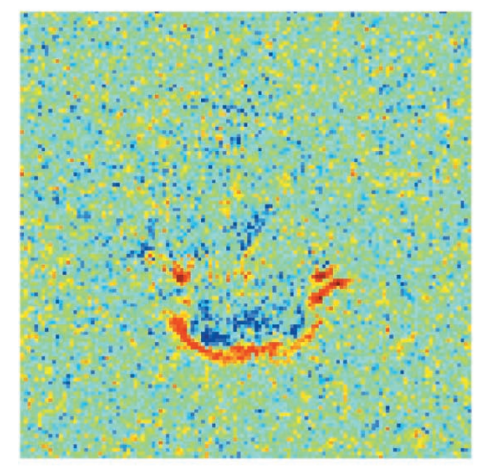

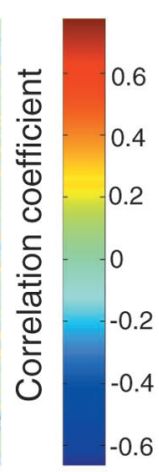

C

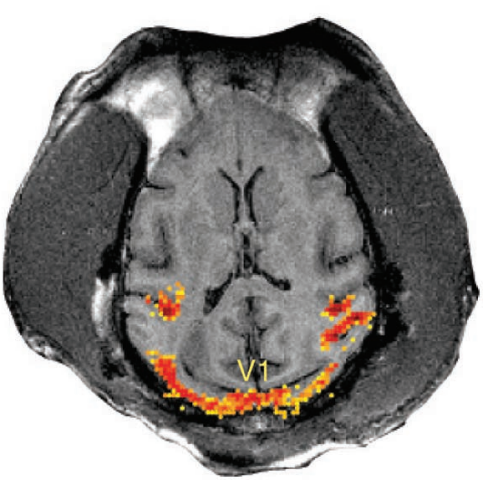

A

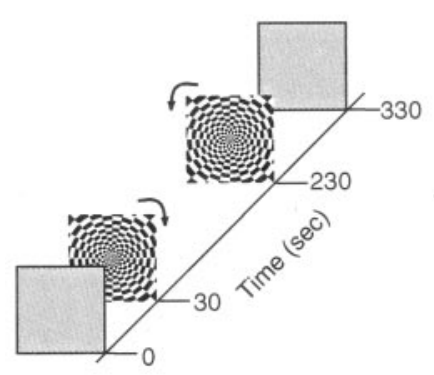

B

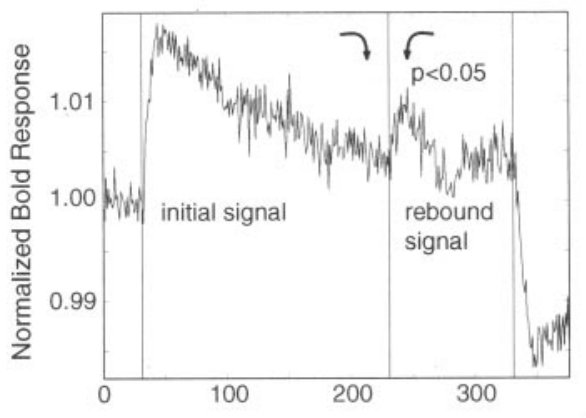

C

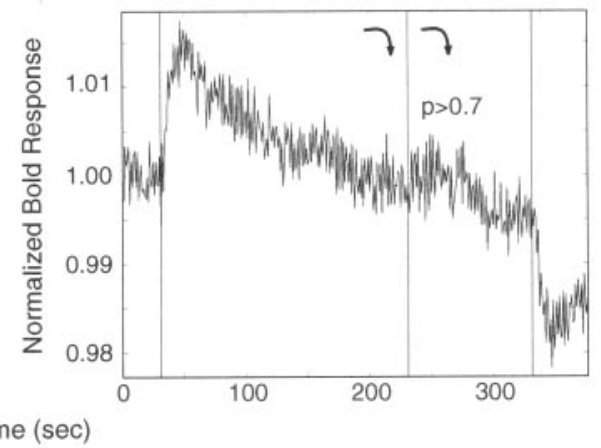

Figure 2. Information about the direction of motion in area V1 in the BOLD signal for a single brain slice. $A$, Visual adaptation protocol. Two to three selected horizontal brain slices were imaged every second with the same individual slice spatial resolution $\left(1 \times 1 \times 2 \mathrm{~mm}^{3}\right)$. Thirty seconds of the OFF-condition were followed by $200 \mathrm{sec}$ of the ON-condition while the polar pattern was rotating continuously in only one of the two motion directions, i.e., either clockwise or counterclockwise. In interleaved trials the direction of motion of the polar was reversed after 200 sec of stimulus onset, and presentation continued for another $100 \mathrm{sec}$. At the point of transition in both cases, the phase of rotation of the polar was reset and was visible as a fast transient. Finally, $48 \mathrm{sec}$ of the OFF-condition was presented. $B$, Average time course of the BOLD signal in area V1 from a single slice (in Fig. $1 C$, 170 voxels highlighted in yellow, 20 repetitions). The signal is normalized to the baseline (dividing by the mean activity during the initial OFF-condition). Therefore, a signal of 1.02 represents an increase of $2 \%$ from baseline. Arrows indicate the direction of motion of the stimulus. The rebound for each stimulation trial was defined as the difference between the mean signal for $30 \mathrm{sec}$ before and after the change in the direction of motion across all activated voxels from V1. The mean of their distribution across identical repetitions is significantly bigger than zero (two-tailed paired $t$ test, $p<0.05$ ), indicating an increase in the BOLD signal after the change in the direction of motion. $C$, Same analysis as $B$ but with no change in the direction of motion. The mean of the distribution is not significantly different from zero (two-tailed paired $t$ test, $p>0.7$ ).

anterior banks of the lunate sulcus, respectively. The fundus of the lunate was not included in either V2 or V3A. Instead, the fundus was analyzed separately in combination with the anterior bank, as a region including both $\mathrm{V} 3$ and V3A (see Fig. 5, V3, $V 3 A$ ). We determined area V4 to be located on the prelunate gyrus between the lunate and superior temporal sulcus, whereas area MT was identified to lie in the posterior bank and fundus of the superior temporal sulcus. Finally, we examined the activation of a region that included both V2 and V3 (see Fig. 5, V2/V3) within the inferior occipital sulcus.

We analyzed the time course of BOLD activity during adaptation to the moving polar stimulus in the above areas. The typical time course of BOLD activity from area V1 is illustrated for a single slice in Figure 2. The BOLD signal rises quickly to a peak after the onset of the stimulus, and then adapts slowly while the polar stimulus is rotating in the initial direction of motion (Fig. 2B). We defined the size of the initial peak in BOLD activation as the initial-response (see Materials and Methods). After the reversal of the direction of motion of the stimulus, a second peak is seen in the BOLD signal that reflects release from adaptation to the initial direction of motion of the polar stimulus. We defined this second peak in BOLD as the rebound-response (Fig. 2B). The existence of the rebound-response demonstrates explicitly that direction of motion information is reflected in the BOLD signal.

Interleaved with the adaptation protocol, we ran a control experiment. In the control, the direction of motion of the visual stimulus did not change. Note that in both experiment and con- 
A



B

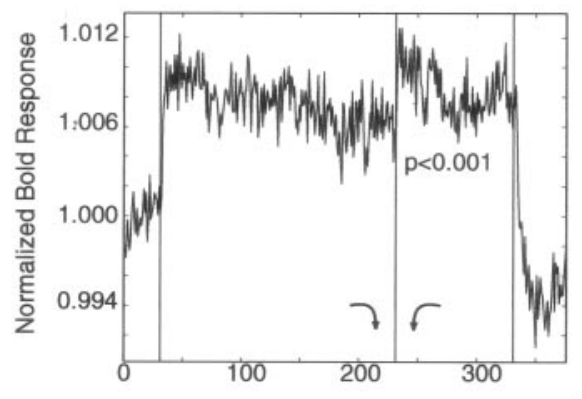

C

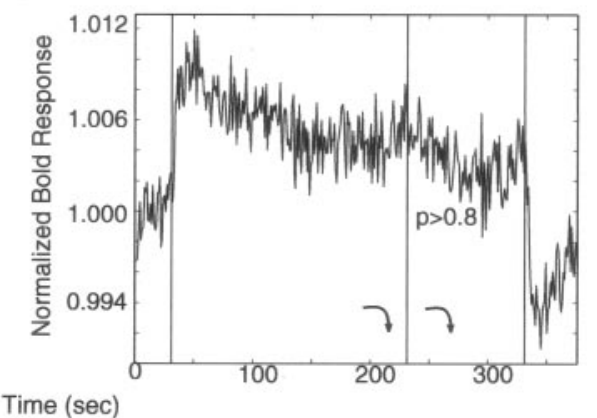

Figure 3. Information about the direction of motion in area MT for a single brain slice (58 voxels). Same experiment and brain slice as that shown in Figure 1, $B$ and $C$. $A-C$, Same analysis as in Figures $1 C$ and 2, $B$ and $C$, respectively. $B$, The mean of the distribution is significantly greater than zero (two-tailed paired $t$ test, $p<10^{-4}$ ) when a change in the direction of rotation occurs. $C$, The mean of the distribution is not significantly different from zero (two-tailed paired $t$ test, $p>0.8$ ) when the direction of rotation remains constant.

trol, the rotating polar was reset to its starting position $200 \mathrm{sec}$ after the onset of the visual stimulus (Fig. 2A) (see Materials and Methods). This resetting could be seen on the monitor in both cases as a brief transient that did not influence significantly the time course of the BOLD, as evidenced by the lack of significant rebound-response in the control experiments (Figs. $2 C, 3 C$, and see Fig. 7A-C).

Visual area MT showed a more pronounced rebound-response than seen in V1 (Fig. 3B). This result agrees with the large proportion of directionally selective cells found in MT and the central role this area plays in the perception of the direction of motion (Maunsell and Van Essen, 1983b; Albright et al., 1984; Newsome et al., 1989; Newsome and Salzman, 1993; Salzman and Newsome, 1994). The mean time course of the BOLD signal averaged over the MT-activated voxels (Fig. $3 A$ ) showed a sharp increase after the onset of the visual stimulus (Fig. $3 B$ ). Subsequently, the signal gradually decreased because of adaptation. Compared with V1, the BOLD signal in area MT reached steady state faster and was more sustained relative to its peak value (Figs. 2C, 3C, 7 ).

In addition to areas V1 and MT (Fig. 4), we also found activation in areas V2, V3, V3A, and V4. We observed a statistically significant rebound-response in all of these cortical regions, reflecting the processing of direction of motion information at the level of the BOLD signal (Fig. 5, right column). The relative strength of the rebound-response versus the initial-response varied across these areas, indicating the difference in processing of motion signals among them.

To compare the results from BOLD with the results from single-cell electrophysiology, we quantified the degree of sensitivity of the BOLD signal to the change in the direction of rotation of the polar stimulus across visual areas (Fig. 6). We defined the BOLD directionality index as the ratio of the rebound to the initial response (see Materials and Methods). A directionality index approximately equal to zero indicates that the BOLD signal from an area is modulated little by changes in the direction of motion, whereas a ratio close to one indicates strong modulation. Area V1 had a BOLD directionality index of 0.33 with a SEM of 0.03 . This index is unexpectedly higher than what may be predicted from the proportion of directionally selective cells found by single-unit recordings in V1, which is $\sim 20 \%$ (see Discussion for more details) (Schiller et al., 1976; De Valois et al., 1982; Albright, 1984; Orban et al., 1986; Hawken et al., 1988;
A

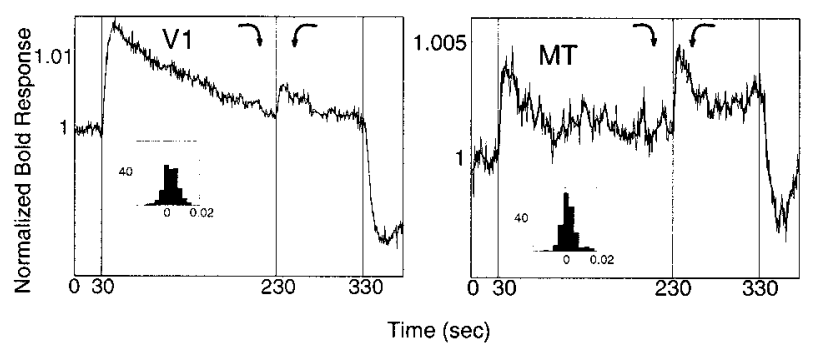

Figure 4. Information about the direction of motion in areas V1 and MT across monkeys. $A$, Mean activity in $\mathrm{V} 1$ across all significant voxels, slices, monkeys, and experimental sessions during visual adaptation experiments (1071 voxels, 10 slices, 4 monkeys, 4 experimental sessions). Histogram inset shows the distribution of the reactivation of the signal (Fig. $2 B$, rebound signal). The reactivation for each stimulation trial was defined as the difference between the mean signal for $30 \mathrm{sec}$ before and after the change in the direction of motion across all activated voxels from V1. A positive reactivation indicates an increase in the signal after the change in the direction of motion. The mean of the distribution is significantly bigger than zero (two-tailed paired $t$ test; $p$ values shown in Figures). Smooth curves represent the mean signal filtered with a digital low-pass Butterworth 8 order filter and cutoff frequency of $0.125 \mathrm{~Hz}$. $B$, Same as $A$ but for area MT (419 voxels, 9 slices, 4 monkeys, 6 experimental sessions). The rebound-response (average response for $30 \mathrm{sec}$ after transient) during the condition in which the direction of the stimulus changed was significantly higher than the control rebound-response in which the direction of the stimulus remained the same (two-tailed paired $t$ test, $p<10^{-6}$ for $\mathrm{V} 1$ and $p<10^{-6}$ for MT).

Chaudhuri and Albright, 1997; Chaudhuri et al., 1997). In area $\mathrm{V} 2$ and the combined region V2/V3, we found the BOLD directional index to be $0.35(\mathrm{SEM}=0.06)$ and $0.37(\mathrm{SEM}=0.04)$, respectively, and the proportion of directional neurons in V2 and V3 to be $\sim 40 \%$ (Foster et al., 1985; Burkhalter and Van Essen, 1986). The BOLD directional index in V3A and the region $\mathrm{V} 3 / \mathrm{V} 3 \mathrm{~A}$ was $0.42(\mathrm{SEM}=0.16)$ and $0.43(\mathrm{SEM}=0.25)$, respectively. In V3A the proportion of directionally selective cells is similar to that in V1 (Gaska et al., 1988).

Areas MT and V4 had higher BOLD directionality indices than those found in earlier visual areas V1, V2, V3, and V3A. Specifically, we found indices of $1(\mathrm{SEM}=0.29)$ and $0.84(\mathrm{SEM}=0.15)$ for V4 and MT, respectively. The strong directionality index in MT is consistent with single-cell studies, which report that a large proportion of MT neurons are directionally selective (Maunsell 

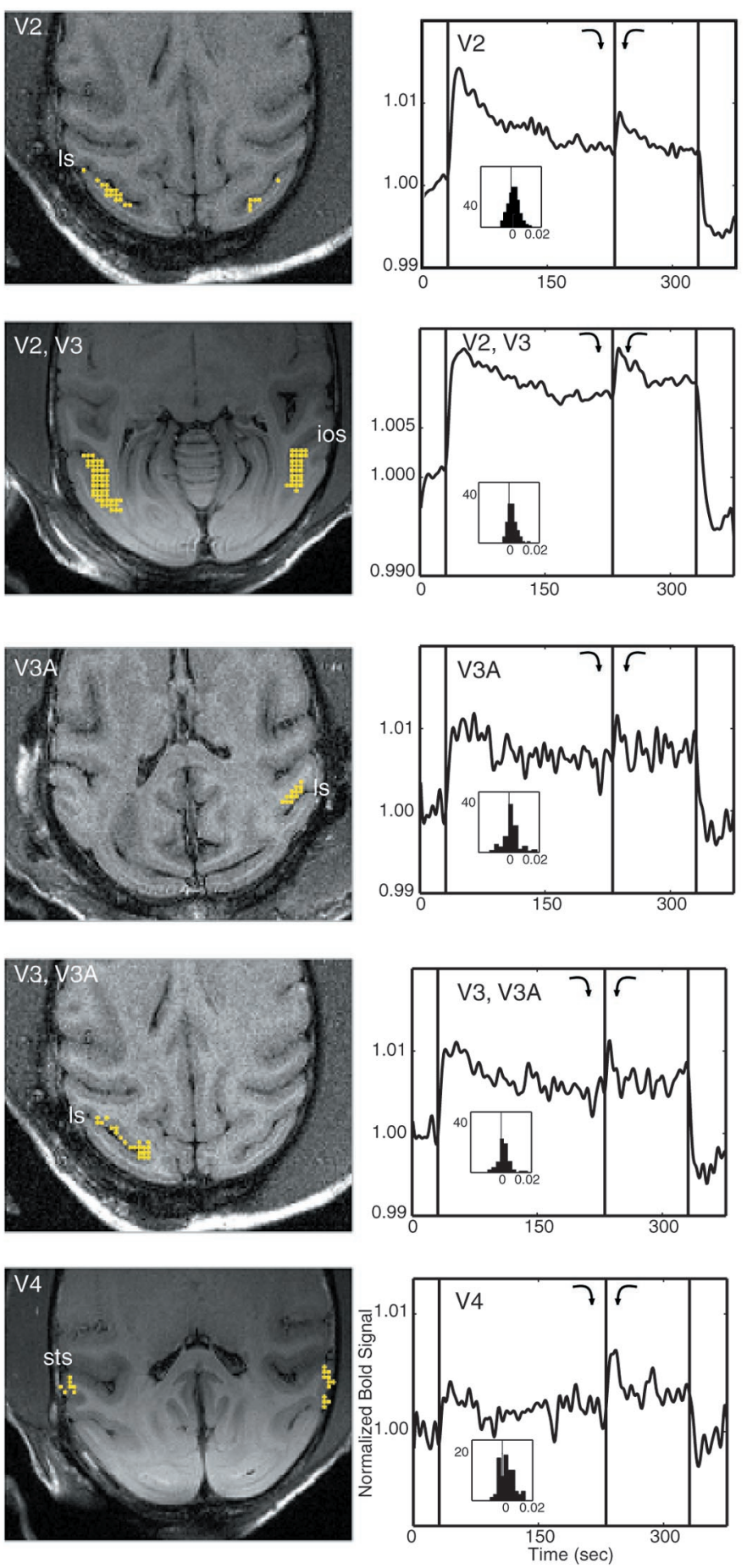

Figure 5. Information about the direction of motion in areas V2, V3, $\mathrm{V} 3 \mathrm{~A}$, and V4. Left column shows the area of interest, marked in yellow, on a single horizontal slice of an individual monkey during one experimental session. Right column (same notations as in Fig. 4) shows the average activities of the different visual areas. This activity is the mean across all significant voxels in all slices, monkeys, and experimental sessions belonging to a particular visual area. Histogram insets have the same notation as in Figure 4. Voxels belonging to area V2 were identified within the posterior bank of the lunate sulcus ( $l s)$ excluding the fundus. The time course of the mean BOLD activity was computed from a total of 157 voxels, six slices, three monkeys, and five experimental sessions. Voxels belonging to area V2/V3 were typically identified within the inferior occipital sulcus (ios). The time course of the mean BOLD activity was computed from a total of 680 voxels, six slices, four monkeys, and four experimental sessions. Voxels belonging to area V3A were identified and Van Essen, 1983a; Albright et al., 1984). However, the strong rebound-response that we found in the BOLD signal of area V4 after adaptation was unexpected, because in contrast to MT, most of the cells in area V4 are not directionally selective. Desimone and Schein (1987) report that the degree of directionality at the level of single cells in V4 is similar to the one found in V1. One qualification of this observation is that the BOLD directionality index we computed for area V4 is not as robust as that of the other visual areas. Activation of area V4 during our experiments was more erratic, possibly because of the particular properties of the stimulus used and/or as a result of anesthesia. Future experiments in alert animals will shed further light in this direction.

\section{Time course of BOLD signal during adaptation in areas V1, V2/V3, and MT}

Activation in areas V1, V2/V3, and MT was robust and reliably observed, which enabled us to quantify the dynamics of adaptation of the BOLD signal in these areas. We analyzed the data collected during control stimulation trials when the direction of motion of the stimulus did not change. An exponential function was fitted to the tail of the BOLD time course giving estimates of the rate of adaptation $(\tau)$ and the steady-state level attained by the BOLD signal (see Materials and Methods).

We found that the adaptation dynamics of the BOLD signal are not the same throughout the various visual areas, presumably reflecting different underlying adaptation dynamics of the corresponding neuronal ensembles. The BOLD signal in MT adapts faster than in V1 (Fig. 7A). Moreover, the activity of MT at steady state is sustained at a higher level than in V1 (Fig. $7 F$ ), despite the fact that the initial-response peak in the BOLD signal is higher in V1 (Fig. $7 G$ ). The adaptation properties of the BOLD signal in $\mathrm{V} 2 / \mathrm{V} 3$ were similar to those in MT, i.e., fast rate of adaptation and high steady state (Fig. $7 D, E$ ).

The size of the initial-response peak of the BOLD signal is similar in areas V1 and V2/V3 but much smaller in area MT. The reason for this is not obvious, but it may reflect the fraction of cells that initially respond to the stimulus in the respective areas as well as the respective cell density. However, the peak responses are similar in size between V2/V3 and V1 despite the fact that there are twice as many neurons per unit volume in the latter (Rockel et al., 1980; Peters, 1987).

\section{DISCUSSION}

The results presented in this paper show that a distributed network of visual areas in the monkey contains information about the direction of motion of a stimulus, in agreement with previous single-unit electrophysiology studies. Specifically, the BOLD signal in areas $\mathrm{V} 1, \mathrm{~V} 2, \mathrm{~V} 3, \mathrm{~V} 3 \mathrm{~A}, \mathrm{~V} 4$, and $\mathrm{MT}$ reflects the processing

within the anterior bank of the $l s$, excluding the fundus. The time course of the mean BOLD activity was computed from a total of 54 voxels, five slices, four monkeys, and four experimental sessions. Voxels belonging to V3/V3A were identified within the anterior bank of the $l s$, including the fundus. The time course of the mean BOLD activity was computed from a total of 170 voxels, five slices, four monkeys, and four experimental sessions. Voxels belonging to area $\mathrm{V} 4$ were identified on the prelunate gyrus. The time course of the mean BOLD activity was computed from a total of 38 voxels, three slices, two monkeys, and three experimental sessions. The reboundresponse (average response for $30 \mathrm{sec}$ after transient) during the condition in which the direction of the stimulus changed was significantly higher than the control rebound-response, where the direction of the stimulus remained the same (two-tailed paired $t$ test; $p<10^{-3}$ for V2; $p<10^{-9}$ for V2/V3; $p<0.05$ for V3A; $p<10^{-3}$ for V3/V3A; $p<0.001$ for V4). 


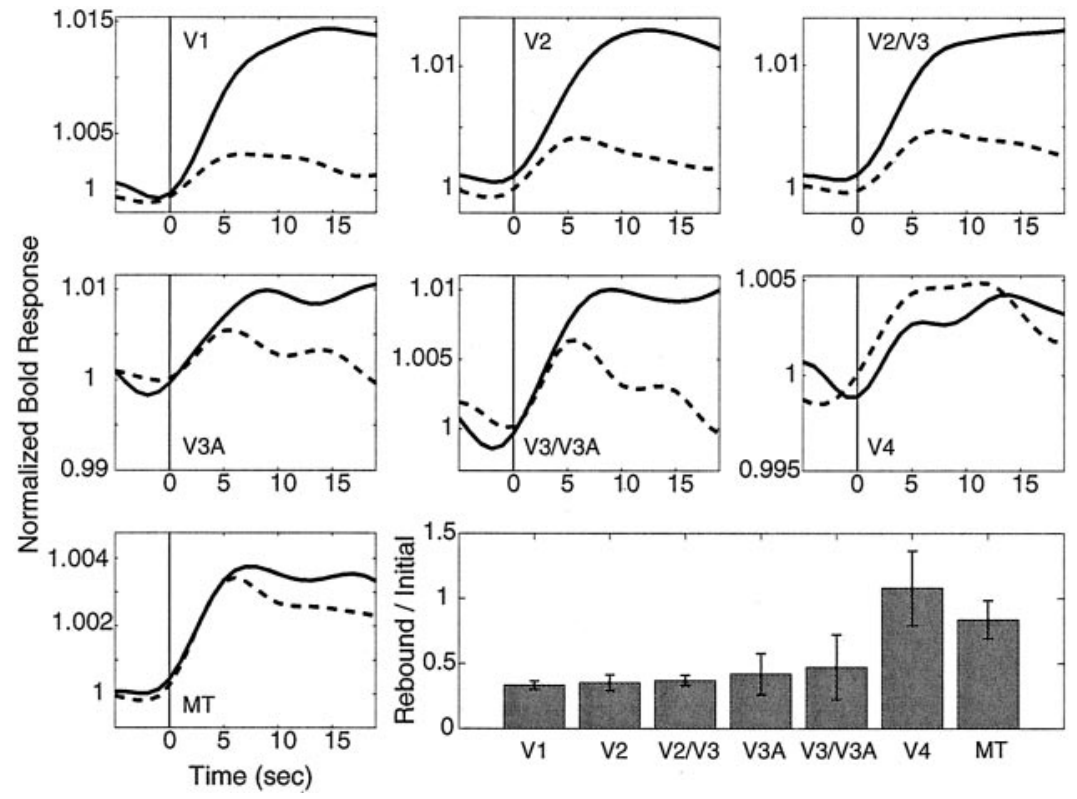

Figure 6. Information about direction of motion across different visual areas. The mean initial and rebound filtered responses (digital low-pass Butterworth 8 order filter and cutoff frequency $0.125 \mathrm{~Hz}$ ) for different visual areas are plotted as continuous and dotted lines, respectively. These signals represent the mean across all slices and experimental sessions. If the mean activity (from 11 to $20 \mathrm{sec}$ after stimulus onset) from an individual slice for a particular visual area was $<2$ SDs above the mean of the baseline (activity during $30 \mathrm{sec}$ of blank screen condition), then this response was excluded from the analysis. Histogram, BOLD directionality indices ( $\rho=$ rebound-response/initial-response) (see Materials and Methods and Fig. $2 B$ ). For V1 mean $\rho=$ $0.33(\mathrm{SEM}=0.03)$, for $\mathrm{V} 2$ mean $\rho=0.35(\mathrm{SEM}=0.06)$, for $\mathrm{V} 2 / \mathrm{V} 3$ mean $\rho=0.37(\mathrm{SEM}=0.04)$, for V3A mean $\rho=0.42$ $(\mathrm{SEM}=0.16)$, for $\mathrm{V} 3 / \mathrm{V} 3 \mathrm{~A}$ mean $\rho=0.43(\mathrm{SEM}=0.25)$, for V4 mean $\rho=1($ SEM $=0.29)$, and for MT mean $\rho=0.84$ $(\mathrm{SEM}=0.15)$. The BOLD directional index in $\mathrm{V} 1$ was significantly smaller than the index of both MT and V4 (two-tailed paired $t$ test, $p<0.001$ for V1, MT comparison; two-tailed paired $t$ test, $p<0.001$ for V1, V4 comparison). The BOLD directional indices for V4 and MT were not significantly different (two-tailed paired $t$ test, $p>0.05$ ). of information about the direction of motion. Previous studies have provided indirect evidence of directional selective interactions in human area MT, using fMRI measurements of BOLD activity during the motion aftereffect (Tootell et al., 1995; He et al., 1998). In another study, Heeger and colleagues (1999) showed the existence of motion opponent mechanisms in human area MT and, by inference, directionally selective mechanisms. Here we provide direct evidence that direction of motion processing is reflected in the BOLD signal in several visual areas and the first evidence to date for the presence of such directionally selective BOLD signal in early visual areas, such as V1. This result is not surprising, given the existence of directionally selective cells found particularly in layers 6 and 4b of V1 (Hawken et al., 1988).

Moreover, we have characterized the dynamics of adaptation of the BOLD signal to unidirectionally rotating stimuli for different visual areas of the macaque. This analysis sheds new light on the emergent properties of the neuronal ensembles in these areas, given that the BOLD signal is thought to reflect brain activity at the level of neuronal populations. We found that the dynamics of adaptation of the BOLD signal were different across various visual areas of the macaque cortex. In particular, the BOLD signal from MT and V2/V3 adapted faster than the BOLD signal from V1 by more than an order of magnitude. A network of neurons that exhibits faster adaptation to the statistical structure of its environment is more efficient in encoding the input in terms of both energy consumption and increased sensitivity to detect changes.

Another difference among these visual areas is the steady state attained by the BOLD signal during the course of adaptation. Despite the fact that the initial transient is higher in V1 than in MT, the steady-state level of the signal is higher in MT than in V1. Similar to the signal in MT, the signal in V2/V3 also attains a high steady state. These results provide additional evidence that the processing of the sensory input in MT and V2/V3 is quite different from that in V1. It is currently thought that the BOLD signal is correlated more with synaptic events than action potentials, and thus the dissimilarities in adaptation among the visual areas likely reflect differences in processing information at the level of synapses (Nunez and Silberstein, 2000; Logothetis et al., 2001).
It is of particular importance that we now have data about the direction of motion information from both fMRI and single cells from the same species. This gives us the opportunity to make semiquantitative comparisons between known results from electrophysiology and the BOLD signal. We find that the BOLD directional index in visual areas such as V1 and V4 was higher than might be expected on the basis of the proportion of cells that are directionally selective in these areas. It is estimated that $\sim 20 \%$ of cells in V1 are directionally selective (Schiller et al., 1976; De Valois et al., 1982; Albright, 1984; Orban et al., 1986; Hawken et al., 1988; Chaudhuri and Albright, 1997; Chaudhuri et al., 1997). The rotating polar stimulus is expected to activate almost all the cells that are typically studied in V1, albeit half of the directionally selective ones. Therefore, $\sim 90 \%$ of the V1 neurons will be activated initially after the polar starts rotating in a single direction. On the other hand, the rebound-response will reflect only half of the directionally selective cells, i.e., $\sim 10 \%$. Accordingly, the BOLD directional index should be $\sim 0.11$ $(10 / 90)$, which is much lower than the ratio of 0.33 that we calculated (Fig. 4B). In area V4 the discrepancy is even larger because the BOLD directionality index is close to 1 , despite the fact that the percentage of directional cells in V4 is close to that of V1. In V4 we did not get responses as robust as those in other areas such as V1 and MT. Nevertheless, when the activity in area V4 was significantly different from background, a change in the direction of motion of the stimulus induced a statistically significant rebound-response with a normalized magnitude that was similar to the normalized magnitude of the response seen in MT.

We propose a hypothesis, which is based on the numerous connections that exist among neurons within and between brain areas, that could account for the apparent difference between the single-unit electrophysiology and BOLD results. This takes advantage of the fact that neuronal directional selectivity is a function of the state of adaptation. Specifically, neurons that under classical investigation may not be directionally selective can manifest directional selectivity after adapting to directional stimuli. Consider a network of neurons where directionally selective cells activate other cells, so that the latter group of neurons is classically nondirectionally selective. This can be simply achieved if heterogeneous populations of directionally selective cells con- 
A

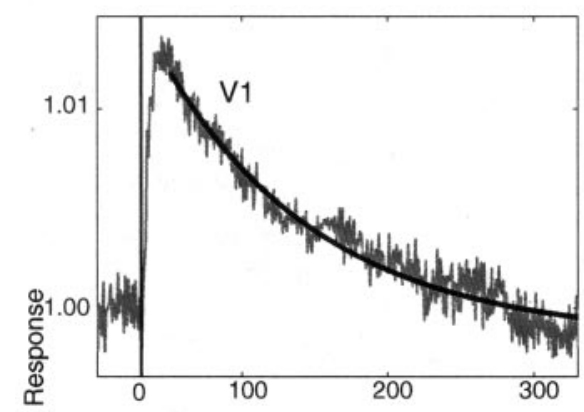

D

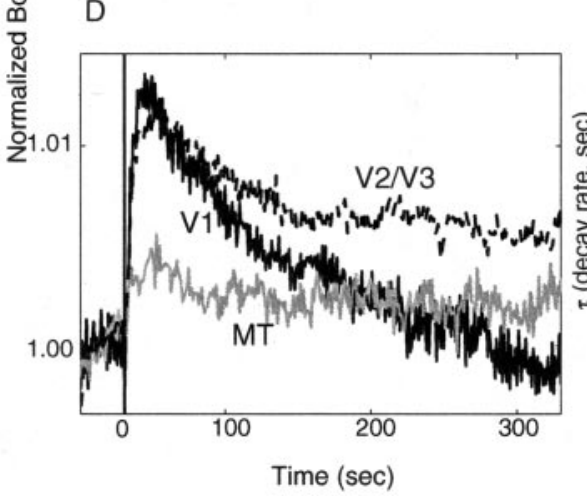

B

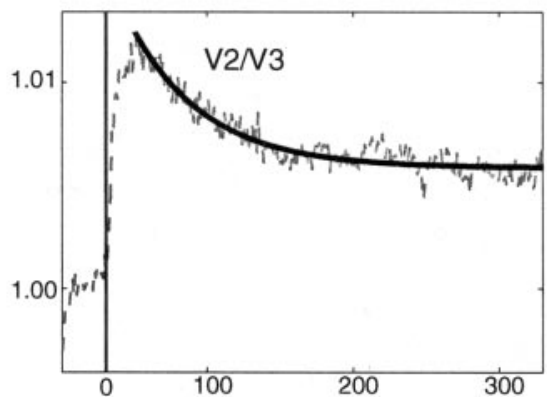

C

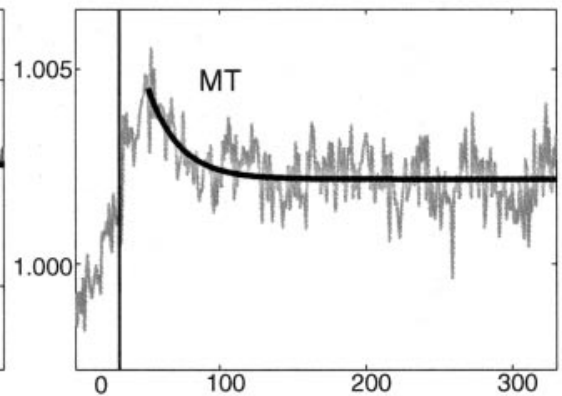

$\mathrm{E}$

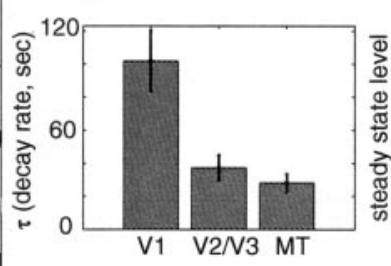

$\mathrm{F}$

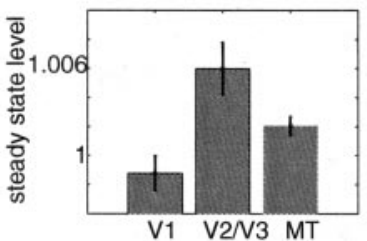

G

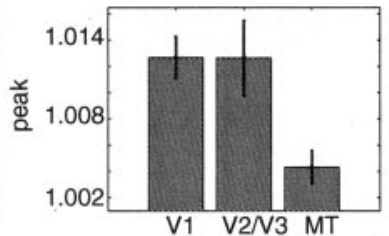

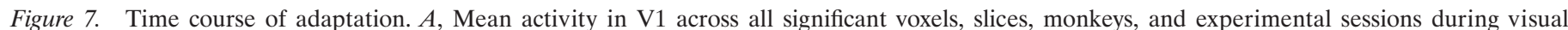

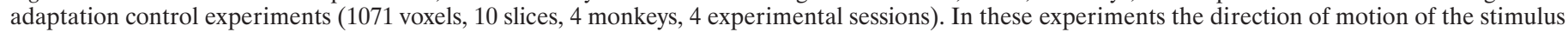

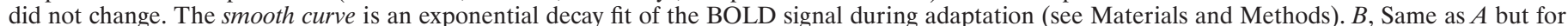

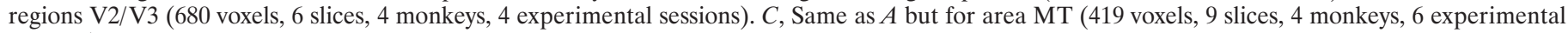

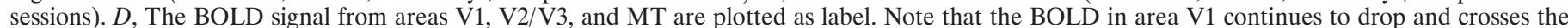

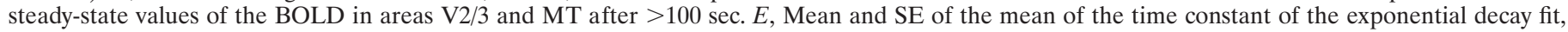

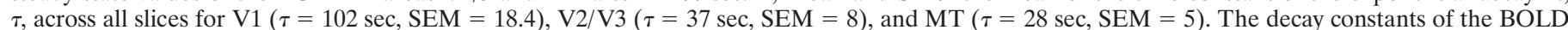

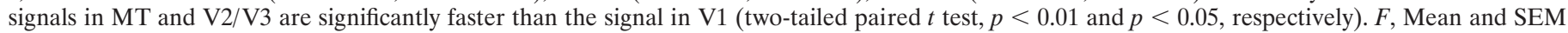

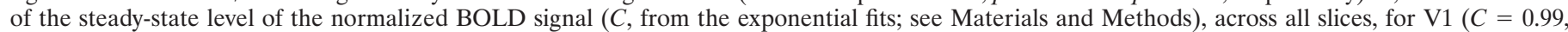

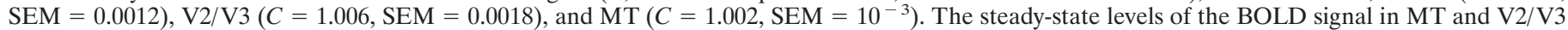

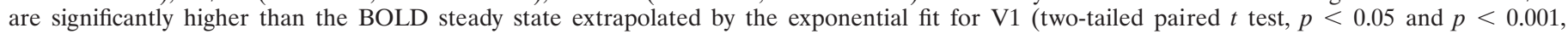

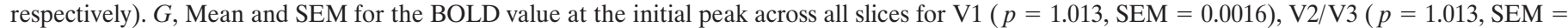
$0.0028)$, and $\operatorname{MT}(p=1.004, \operatorname{SEM}=0.0012)$.

verge to provide balanced input to the nondirectionally selective neurons. The directionally selective cells will show robust activity only when the preferred stimulus is presented in the visual field. On the other hand, the classically "nondirectionally selective" cells will be activated when stimulated with either direction of motion since they received balanced directionally selective input. Note that after the network adapts to a particular direction of motion of the visual stimulus, a change in the direction of motion alters the balance of the directionally selective input to the nondirectionally selective neurons and results in increased neuronal activity, thereby contributing to the rebound signal. Therefore, one possible source for the higher than expected BOLD directional index in visual areas such as V1 could be the activation of classically nondirectionally selective neurons that receive input from directionally selective cells, e.g., from MT cells (Rockland and Knutson, 2000). Recent work showing that the orientation tuning of complex cells in macaque V1 is affected by the orientation of an adapting stimulus (Muller et al., 1999; Dragoi et al., 2000) provides evidence supporting a mechanism similar to the one postulated by our hypothesis. Note that our hypothesis is only a tentative suggestion. There are several other schemes that could explain our observations, such as local interactions between populations of neurons tuned to opposite directions of motion.
Recently, fMRI and electrophysiological signals were measured simultaneously and compared to study their relationship (Logothetis et al., 2001). Logothetis and colleagues (2001) found that the BOLD signal was more correlated with the local field potential than with the multiunit spiking activity. Local field potentials are thought to reflect mostly somatodendritic events and therefore, presumably, neuronal inputs. Our result, of stronger than expected directional signal in certain visual areas, may reflect directionally selective input to these areas that are not revealed with classical electrophysiology experiments, which focus mostly on neuronal outputs.

The comparison that we attempt between the magnitude of the BOLD directionally selective signal and single-unit recordings is at best semiquantitative, particularly because we use a different visual stimulation paradigm. Future studies in which BOLD and neuronal activity are recorded simultaneously during adaptation will delineate further the mechanism underlying the directionally selective BOLD signal.

The role of adaptation in neuronal computations is a timehonored principle of single-cell physiology (Barlow, 1972). Here we suggest that connectivity between neurons in a distributed hierarchical network may mediate a change of neuronal specificity in early visual areas as a function of adaptation to high-level 
visual attributes computed in higher areas. Neurons in early visual areas, thought to lack information about certain attributes of the visual scene when tested classically, might nevertheless be able to adapt specifically to those attributes; thereby, neurons in early visual areas may be recruited to encode changes along specific stimulus dimensions that carry high information content.

\section{REFERENCES}

Albright TD (1984) Direction and orientation selectivity of neurons in visual area MT of the macaque. J Neurophysiol 52:1106-1130.

Albright TD, Desimone R, Gross CG (1984) Columnar organization of directionally selective cells in visual area MT of the macaque. J Neurophysiol 51:16-31.

Barlow HB (1972) Single units and sensation: a neuron doctrine for perceptual psychology? Perception 1:371-394.

Barlow HB, Hill RM (1963) Evidence for a physiological explanation of the waterfall phenomenon and figural after effects. Nature 200:1345-1347.

Burkhalter A, Van Essen DC (1986) Processing of color form and disparity information in visual areas VP and V2 of ventral extrastriate cortex in the macaque monkey macaca-fascicularis. J Neurosci 6:2327-2351.

Chaudhuri A, Albright TD (1997) Neuronal responses to edges defined by luminance vs. temporal texture in macaque area V1. Vis Neurosci 14:949-962.

Chaudhuri A, Nissanov J, Larocque S, Rioux L (1997) Dual activity maps in primate visual cortex produced by different temporal patterns of zif268 mRNA and protein expression. Proc Natl Acad Sci USA 94:2671-2675.

Desimone R, Schein SJ (1987) Visual properties of neurons in area V4 of the macaque: sensitivity to stimulus form. J Neurophysiol $57: 835-868$.

Desimone R, Ungerleider LG (1986) Multiple visual areas in the caudal superior temporal sulcus of the macaque. J Comp Neurol 248:164-189.

De Valois RL, Yund EW, Hepler N (1982) The orientation and direction selectivity of cells in macaque visual cortex. Vision Res 22:531-544.

Dragoi V, Sharma J, Sur M (2000) Adaptation-induced plasticity of orientation tuning in adult visual cortex. Neuron 28:287-298.

Foster KH, Gaska JP, Nagler M, Pollen DA (1985) Spatial and temporal frequency selectivity of neurons in visual cortical areas V1 and V2 of the macaque monkey. J Physiol (Lond) 365: 331-364.

Gaska JP, Jacobson LD, Pollen DA (1988) Spatial and temporal frequency selectivity of neurons in visual cortical area V3A of the macaque monkey. Vision Res 28:1179-1191.

Gattass R, Gross CG, Sandell JH (1981) Visual topography of V2 in the Macaque. J Comp Neurol 201:519-539.

Gattass R, Sousa AP, Gross CG (1988) Visuotopic organization and extent of V3 and V4 of the macaque. J Neurosci 8:1831-1845.

Grill-Spector K, Kushnir T, Edelman S, Avidan G, Itzchak Y, Malach R (1999) Differential processing of objects under various viewing conditions in the human lateral occipital complex. Neuron 24:187-203.

Hawken MJ, Parker AJ, Lund JS (1988) Laminar organization and contrast sensitivity of direction-selective cells in the striate cortex of the Old World monkey. J Neurosci 8:3541-3548.

He S, Cohen ER, Hu X (1998) Close correlation between activity in brain area MT/V5 and the perception of a visual motion aftereffect. Curr Biol 8:1215-1218.

Heeger DJ, Boynton GM, Demb JB, Seidemann E, Newsome WT (1999) Motion opponency in visual cortex. J Neurosci 19:7162-7174.

Ibbotson MR, Clifford CW, Mark RF (1998) Adaptation to visual motion in directional neurons of the nucleus of the optic tract. J Neurophysiol 79:1481-1493.
Kourtzi Z, Kanwisher N (2000) Cortical regions involved in perceiving object shape. J Neurosci 20:3310-3318.

Lisberger SG, Movshon JA (1999) Visual motion analysis for pursuit eye movements in area MT of macaque monkeys. J Neurosci 19:2224-2246.

Logothetis NK, Guggenberger H, Peled S, Pauls J (1999) Functional imaging of the monkey brain. Nat Neurosci 2:555-562.

Logothetis NK, Pauls J, Augath M, Trinath T, Oeltermann A (2001) Neurophysiological investigation of the basis of the fMRI signal. Nature 412:150-157.

Maddess T, McCourt ME, Blakeslee B, Cunningham RB (1988) Factors governing the adaptation of cells in area-17 of the cat visual cortex. Biol Cybern 59:229-236.

Mather G, Verstraten FAJ, Anstis S (1998) The motion aftereffect. A modern perspective. Cambridge, MA: MIT.

Maunsell JHR, Newsome WT (1987) Visual processing in monkey extrastriate cortex. Annu Rev Neurosci 10:363-401.

Maunsell JHR, Van Essen DC (1983a) Functional properties of neurons in middle temporal visual area of the macaque monkey: I. Selectivity for stimulus direction, speed, and orientation. J Neurophysiol 49:1127-1147.

Maunsell JHR, Van Essen DC (1983b) The connections of the middle temporal visual area (MT) and their relationship to a cortical hierarchy in the macaque monkey. J Neurosci 3:2563-2586.

Muller JR, Metha AB, Krauskopf J, Lennie P (1999) Rapid adaptation in visual cortex to the structure of images. Science 285:1405-1408.

Newsome WT, Salzman CD (1993) The neuronal basis of motion perception. Ciba Found Symp 174:217-230.

Newsome WT, Britten KH, Movshon JA (1989) Neuronal correlates of a perceptual decision. Nature 341:52-54.

Nunez PL, Silberstein RB (2000) On the relationship of synaptic activity to macroscopic measurements: does co-registration of EEG with fMRI make sense? Brain Topogr 13:79-96.

Orban GA, Kennedy H, Bullier J (1986) Velocity sensitivity and direction selectivity of neurons in areas V1 and V2 of the monkey: influence of eccentricity. J Neurophysiol 56:462-480.

Oyster CW, Takahashi E, Collewijn H (1972) Direction-selective retinal ganglion cells and control of optokinetic nystagmus in the rabbit. Vision Res 12:183-193.

Parker AJ, Newsome WT (1998) Sense and the single neuron: probing the physiology of perception. Annu Rev Neurosci 21:227-277.

Peters A (1987) Number of neurons and synapses in primary visual cortex. In: Cerebral cortex (Jones EG, Peters A, eds), pp 267-294. New York: Plenum.

Rockel AJ, Hiorns RW, Powell TP (1980) The basic uniformity in structure of the neocortex. Brain 103:221-244.

Rockland KS, Knutson T (2000) Feedback connections from area MT of the squirrel monkey to areas V1 and V2. J Comp Neurol 425:345-368.

Salzman CD, Newsome WT (1994) Neural mechanisms for forming a perceptual decision. Science 264:231-237.

Schiller PH, Finlay BL, Volman SF (1976) Quantitative studies of singlecell properties in monkey striate cortex. V. Multivariate statistical analyses and models. J Neurophysiol 39:1362-1374.

Smirnakis SM, Berry MJ, Warland DK, Bialek W, Meister M (1997) Adaptation of retinal processing to image contrast and spatial scale. Nature 386:69-73.

Tootell RBH, Reppas JB, Dale AM, Look RB, Sereno MI, Malach R, Brady TJ, Rosen BR (1995) Visual motion aftereffect in human cortical area MT revealed by functional magnetic resonance imaging. Nature 375:139-141.

Vautin RG, Berkley MA (1977) Responses of single cells in cat visual cortex to prolonged stimulus movement: neural correlates of visual aftereffects. J Neurophysiol 40:1051-1065.

von der Heydt R, Hanny P, Adorjani C (1978) Movement aftereffects in the visual cortex. Arch Ital Biol 116:248-254.

Wohlgemuth A (1911) On the aftereffect of seen movement. Br J Psychol Monograph Suppl 1:1-117. 